\title{
Quantum transport in defective phosphorene nanoribbons: Effects of atomic vacancies
}

\author{
L. L. $\mathrm{Li}^{1,2, *}$ and F. M. Peeters ${ }^{1, \dagger}$ \\ ${ }^{1}$ Department of Physics, University of Antwerp, Groenenborgerlaan 171, B-2020 Antwerpen, Belgium \\ ${ }^{2}$ Key Laboratory of Materials Physics, Institute of Solid State Physics, Chinese Academy of Sciences, Hefei 230031, China
}

(Received 2 January 2018; published 13 February 2018)

\begin{abstract}
Defects are almost inevitably present in realistic materials and defective materials are expected to exhibit very different properties than their nondefective (perfect) counterparts. Here, using a combination of the tight-binding approach and the scattering matrix formalism, we investigate the electronic transport properties of defective phosphorene nanoribbons (PNRs) containing atomic vacancies. We find that for both armchair PNRs (APNRs) and zigzag PNRs (ZPNRs), single vacancies can create quasilocalized states, which can affect their conductance. With increasing vacancy concentration, three different transport regimes are identified: ballistic, diffusive, and Anderson localized ones. In particular, ZPNRs that are known to be metallic due to the presence of edge states become semiconducting: edge conductance vanishes and transport gap appears due to Anderson localization. Moreover, we find that for a fixed vacancy concentration, both APNRs and ZPNRs of narrower width and/or longer length are more sensitive to vacancy disorder than their wider and/or shorter counterparts, and that for the same ribbon length and width, ZPNRs are more sensitive to vacancy disorder than APNRs.
\end{abstract}

DOI: 10.1103/PhysRevB.97.075414

\section{INTRODUCTION}

Phosphorene, a single layer of black phosphorus (BP), is a relatively new two-dimensional (2D) material [1-4], which has drawn a lot of attention. Bulk BP is a layered material in which individual phosphorene layers are vertically coupled via the van der Waals interaction. Because of this weak interlayer interaction, phosphorene has been successfully obtained by the method of exfoliation [5-9]. Inside a single layer, each phosphorus $(\mathrm{P})$ atom is bonded with three nearest $\mathrm{P}$ atoms via $s p^{3}$ hybridization, thereby forming a puckered honeycomb lattice. Due to this unique lattice structure, phosphorene exhibits a highly anisotropic band structure, which gives rise to highly anisotropic single-particle as well as many-body (e.g., plasmonic and excitonic) properties [10-20], which are atypical for most 2D materials such as graphene and monolayer $\mathrm{MoS}_{2}$. Moreover, theoretical calculations based on density-functional theory (DFT) demonstrate that phosphorene has the combined properties of a considerably large band gap $(\sim 1.5 \mathrm{eV})$ and a relatively high carrier mobility $\left(\sim 10^{3} \mathrm{~cm}^{2} \mathrm{~V}^{-1} \mathrm{~s}^{-1}\right)$ [11], which indicates that this material is very promising for electronic applications in, e.g., field-effect transistors.

Recently, phosphorene nanostructures (such as nanodots, nanorings, and nanoribbons) have drawn a lot of attention. Among them, phosphorene nanoribbons (PNRs) are of particular interest. PNRs can, in principle, be fabricated with lithography and plasma etching of bulk phosphorene, similar to graphene nanoribbons (GNRs) that were previously fabricated from bulk graphene [21,22]. These one-dimensional (1D) nanoribbons show not only the usual confinement effect but also peculiar edge effects, both of which play an important role

\footnotetext{
*longlong.li@uantwerpen.be

†francois.peeters@uantwerpen.be
}

in their electronic, optical, magnetic, and transport properties. Taking PNRs as an example, it was theoretically found that (i) normal armchair PNRs (APNRs) exhibit a $1 / W^{2}$ dependence of the band gap on the ribbon width $W$, while normal zigzag PNRs (ZPNRs) show a $1 / W$ scaling for the band gap [23]; (ii) pristine APNRs are semiconducting, while pristine ZPNRs are metallic unless they undergo an edge reconstruction or passivation [24]; (iii) normal ZPNRs have quasiflat bands associated with edge states [25], while normal APNRs have no such edge bands; (iv) pristine ZPNRs prefer a ground state with ferromagnetic order in the same edge but antiferromagnetic order between two opposite edges [26]; (v) passivated PNRs show improved thermoelectric properties as compared to their pristine counterparts [27], e.g., the Seebeck coefficient of prinstine ZPNRs can be greatly enhanced by hydrogen passivation; and (vi) skewed PNRs show different interesting properties as compared to their normal counterparts [28], e.g., skewed APNRs have quasiflat edge bands which share the same topological origin as those appearing in normal ZPNRs. In addition to the effects arising from confinement and the particular type of edges, external strain and/or electric field can also have a strong influence on the electronic, optical, and transport properties of PNRs [29-32].

In spite of the fast growing knowledge on perfect PNRs as mentioned above, less attention has been paid to their defective counterparts. At present, point defects in phosphorene and bulk $\mathrm{BP}$ have been investigated in a few theoretical and experimental studies. Recent DFT calculations predicted that (i) various types of atomic vacancies (e.g., mono- and divacancies) can be formed in phosphorene and they have quite different influences on the electronic band structure of phosphorene [33]; (ii) intrinsic point defects like native $\mathrm{P}$ vacancies have moderate formation energy and can be stably present in phosphorene, which could act as shallow acceptors [34]; (iii) extrinsic point defects such as foreign adatoms lead to 
distinct modifications of the electronic and transport properties of phosphorene [35]; and (iv) atomic mono- and divacancies are highly itinerant in phosphorene at low temperatures and show different anisotropic mobility features [36]. Most recently, atomic vacancies were experimentally observed on the surface of bulk BP $[37,38]$, and it was found that such vacancies exhibit strongly anisotropic and highly delocalized resonant states [37]. However, compared to defective phosphorene and bulk BP, far less work $[39,40]$ has been done on defective PNRs within a realistic experimental set-up.

From a practical point of view, when PNRs are fabricated experimentally, they will inevitably have some defects (e.g., edge vacancies produced during lithography or plasma etching process), which will affect the electronic and transport properties of PNRs and thus the performance of PNR-based devices. From a fundamental point of view, it is essential to have a better understanding of the electronic and transport properties of defective PNRs and how to control these properties. More importantly, point defects like atomic vacancies were already experimentally observed on the surface of bulk BP $[37,38]$. These motivate us to investigate theoretically the electronic transport properties of defective PNRs with atomic vacancies. As is known, such point defects are one of the most important lattice imperfections in realistic materials [41]. Moreover, in 2D materials, their atomic-scale thickness is expected to be more beneficial for creating atomic vacancies. For instance, a high concentration of such vacancies (up to $2 \%$ ) was experimentally observed in monolayer $\mathrm{MoS}_{2}$ [42,43]. This concentration could be even higher in phosphorene, due to the much lower formation energy of its atomic vacancies [36].

Our theoretical study is carried out by means of atomistic quantum transport simulations based on the tight-binding (TB) approach and the scattering matrix formalism. Both cases of a single atomic vacancy and a random distribution of atomic vacancies are considered in the present paper. For the case of a single vacancy, we study the effects of vacancy type and its spatial location on the electronic and transport properties of defective PNRs. This is not only realistic (because single atomic vacancies have been experimentally observed within several monolayers of BP [37]), but also can provide basic insights into the effects of more complex atomic vacancies. For the case of a random distribution of vacancies, we study the influence of vacancy concentration on the electronic and transport properties of defective PNRs. Due to the random nature of such vacancies, the electronic and transport properties are calculated and averaged over an ensemble of many different vacancy configurations. Moreover, the effects of ribbon length and width are also studied for defective PNRs.

This paper is organized as follows. In Sec. II, we present the theoretical approach for studying the electronic and transport properties of defective PNRs with atomic vacancies. In Sec. III to Sec. V, the main results are presented and analyzed for the effects of such point vacancies on the electronic and transport properties of PNRs. Finally, we summarize our results and give concluding remarks in Sec. VI.

\section{THEORETICAL APPROACH}

The model system under consideration is composed of two leads and a scattering region, as shown in Fig. 1. The scattering
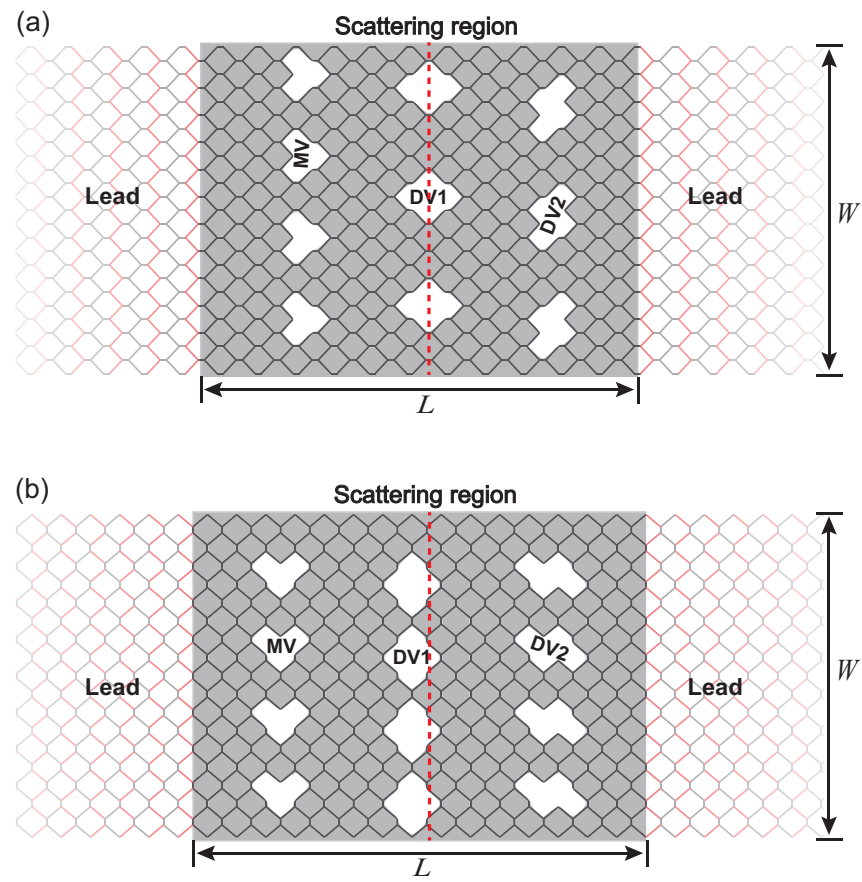

FIG. 1. Model system under consideration: (a) armchair and (b) zigzag PNRs. The system is composed of two semi-infinite leads of width $W$ (light-red shaded region) and a finite scattering region of length $L$ and width $W$ (dark-gray shaded region). Here, three types of atomic vacancies are taken into account, i.e., monovacancy (MV), divacancy of type I (DV1), and divacancy of type II (DV2), which can be located at the edges or in the bulk of the scattering region. Two types of divacancies (DV1 and DV2) are distinguished where DV1 (DV2) keeps (breaks) the sublattice symmetry of the PNR.

region is a finite-size PNR of length $L$ and width $W$, which contains lattice defects induced by atomic vacancies that are located at the edges or in the bulk of the PNR (see Fig. 1). The two leads are assumed to be semi-infinite and perfect PNRs. We use a TB model based on the single $\left(p_{z}\right)$ orbital to describe the low-energy electrons in the PNR. The parameters of this TB model were obtained by fitting its low-energy band structure of phosphorene to the one computed by the DFT-GW approach [44], and it was shown that the fitted TB model can reproduce well the band structure of phosphorene in the low-energy regime as compared to the DFT-GW approach [44]. The TB Hamiltonian of the considered system is

$$
H=\sum_{i} \varepsilon_{i} c_{i}^{\dagger} c_{i}+\sum_{i \neq j} t_{i j} c_{i}^{\dagger} c_{j},
$$

where the summation runs over all lattice sites, $\varepsilon_{i}$ is the on-site energy at site $i, t_{i j}$ is the hopping energy between sites $i$ and $j$, and $c_{i}^{\dagger}\left(c_{j}\right)$ is the creation (annihilation) operator of an electron at site $i(j)$.

In the absence of atomic vacancies, the on-site energies $\varepsilon$ are set to zero for all lattice sites and the hopping energies between two different sites are included up to the fifth nearest neighbors, of which the values are $t_{1}=-1.220 \mathrm{eV}, t_{2}=3.665 \mathrm{eV}, t_{3}=$ $-0.205 \mathrm{eV}, t_{4}=-0.105 \mathrm{eV}$, and $t_{5}=-0.055 \mathrm{eV}$ [44]. In the presence of atomic vacancies, both the on-site and hopping energies are modified. Since a single vacancy can be modeled 
by removing a single atom from the perfect lattice (as shown in Fig. 1), the on-site energy at this atomic site as well as the hopping energies from this atomic site to its nearest neighbors will vanish.

In what follows, we show briefly how to calculate the conductance of the PNR system via the scattering matrix formalism. The detailed description of this formalism can be found in Ref. [45]. Mathematically, the scattering matrix formalism is equivalent to the nonequilibrium Green's function formalism due to the Fisher-Lee relation [46], but it has a simpler structure in its formulation and is more stable in numerical computations [47].

In a two-terminal system composed of two leads and a scattering region, the transport modes (or channels) can be classified into three types: incoming, outgoing, and evanescent ones. The first two carry current corresponding to propagating modes while the last one corresponds to decaying modes. We denote the number of incoming, outgoing, and evanescent modes with $N_{\text {in }}, N_{\text {out }}$, and $N_{\text {ev }}$, respectively, and the corresponding wave functions of these modes with $\psi^{\text {in }}, \psi^{\text {out }}$, and $\psi^{\mathrm{ev}}$. Moreover, we denote the scattering states in the leads and in the scattering region with $\Psi^{L}$ and $\Psi^{S}$, respectively. With these notations and taking the transport direction along the $x$ axis, the scattering state in the leads can be written as [47]

$$
\Psi_{n}^{L}(x)=\psi_{n}^{\mathrm{in}}(x)+\sum_{m=1}^{N_{\text {out }}} S_{m n} \psi_{m}^{\mathrm{out}}(x)+\sum_{k=1}^{N_{\mathrm{ev}}} \mathcal{S}_{k n} \psi_{k}^{\mathrm{ev}}(x),
$$

where $S_{m n}\left(\mathcal{S}_{k n}\right)$ gives the scattering amplitude from an incoming mode $n$ to an outgoing mode $m$ (an evanescent mode $k$ ), both of which are the elements of the scattering matrix ( $S$ matrix). The $S$ matrix can be obtained by matching the wave function in the leads with the one in the scattering region [47], i.e., $\Psi_{n}^{L}\left(x=x_{I}\right)=\Psi_{n}^{S}\left(x=x_{I}\right)$, where $x=x_{I}$ denotes the intersection of the lead and the scattering region.

Once the $S$ matrix is obtained, the conductance of the system at zero temperature can be calculated by using the Landauer formula [45]

$$
G_{a b}=\frac{e^{2}}{h} T_{a b}=\frac{e^{2}}{h} \sum_{n \in a ; m \in b}\left|S_{m n}\right|^{2},
$$

where $a$ and $b$ label the two leads of the system and $T_{a b}$ is the transmission coefficient from lead $a$ to lead $b$. It is clear that the conductance value depends on the number of available transport modes. In the present paper, transport calculations were performed using KWANT [47], while other properties (e.g., density of states) were calculated using PYBINDING [48].

\section{PERFECT PNRS WITHOUT VACANCIES}

Before proceeding with defective PNRs with atomic vacancies, we first have a look at the electronic and transport properties of their perfect counterparts (i.e., without vacancies). In Fig. 2, we show the band structure, density of states (DOS), and conductance of perfect APNR [Figs. 2(a)-2(c)] and of perfect ZPNR [Figs. 2(d)-2(f)]. Here, the ribbon width is set to $W=4 \mathrm{~nm}$ for both APNR and ZPNR. As shown in Figs. 2(a) and 2(d), perfect ZPNR has quasiflat bands
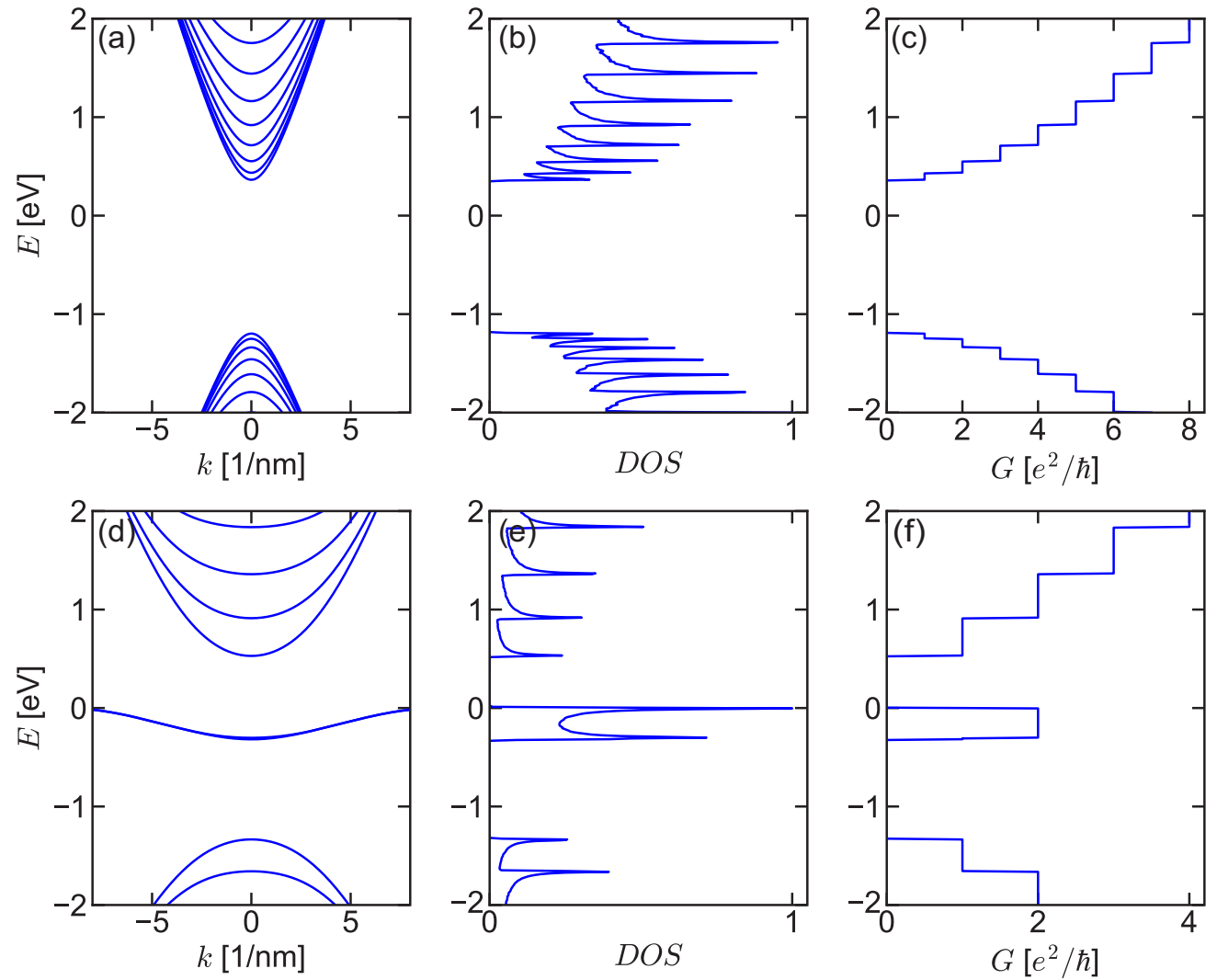

FIG. 2. Band structure, DOS, and conductance of perfect PNRs (without vacancies): (a)-(c) for APNR and (d)-(f) for ZPNR. Here, the ribbon width is set to $W=4 \mathrm{~nm}$ for both APNR and ZPNR. 
within the band gap, which are associated with edge states that are localized at the zigzag boundaries. The presence of such in-gap states indicates a metallic feature of intrinsic (undoped) perfect ZPNRs. However, perfect APNR does not have any states within the band gap, which indicates a semiconducting behavior. There are also many subbands outside the band gap, corresponding to quantized $1 \mathrm{D}$ conduction channels in perfect APNR and ZPNR. The band-structure properties of perfect APNR and ZPNR are also reflected in their DOS spectra. As shown in Figs. 2(b) and 2(e), the DOS of ZPNR has distinct peaks inside the band gap, which are clearly associated with in-gap edge states, while APNR has zero DOS inside the band gap because there are no in-gap (edge) states. In addition, both APNR and ZPNR have a sequence of sharp peaks in their DOS outside the band gap, which correspond to the Van Hove singularities induced by the extrema of their 1D quantized bulk subbands.

The distinctive electronic properties of APNR and ZPNR have a direct consequence on their transport properties. As shown in Figs. 2(c) and 2(f), the conductance of both APNR and ZPNR exhibits quantized plateaus $G=n e^{2} / h$ with $n$ being a positive integer given by the number of available transport channels (modes) for a given energy $E$. When $E$ is located within the band gap around zero energy, the conductance of ZPNR has a quantized plateau of value $G=2 e^{2} / h$, which is clearly induced by edge-propagating modes along the zigzag boundaries. However, for the same $E$, APNR has no such quantized plateau in the conductance because there are no such (edge) propagating modes along the armchair boundaries. Furthermore, we find that the conductance of both APNR and ZPNR is not symmetric with respect to $E=0$ (i.e., it exhibits an electron-hole asymmetry). Our results for the conductance of perfect ZPNR agree with those obtained in previous works $[31,32]$.

\section{EFFECTS OF SINGLE VACANCY}

Now we consider defective PNRs with a single atomic vacancy. We study how such a point defect affects the electronic and transport properties of PNRs. This is not only realistic (because single atomic vacancies were experimentally observed within few layers of BP [37]), but also can provide basic insights into the understanding of the effects of more complex distributions of atomic vacancies.

We first study the effect of the type of vacancy on the DOS and conductance of defective APNR and ZPNR. Three types of single vacancies are considered: (i) monovacancy (MV), (ii) di-vacancy of type I (DV1), and (iii) di-vacancy of type II (DV2). For simplicity, we assume MV, DV1, and DV2 to be located in the center of the scattering region. Notice that two types of divacancies (DV1 and DV2) are distinguished where DV1 (DV2) preserves (breaks) the sublattice symmetry of PNR. Here, the sublattice symmetry (asymmetry) is defined in terms of the number of different sublattice atoms in the PNR. Although the unit cell of phosphorene has four sublattice atoms, it was shown [25] that in the TB model they can be reduced to two inequivalent ones (labeled $A$ and $B$ ) in the unit cell due to the $D_{2 h}$ point group invariance. For convenience, we use $N_{A}$ and $N_{B}$ to denote the number of atoms in sublattices $A$ and $B$ of PNR. In the absence of vacancies, we have
$N_{A}=N_{B}$ and in the presence of vacancies, we state that the sublattice symmetry is preserved (broken) if $N_{A}=N_{B}$ $\left(N_{A} \neq N_{B}\right)$. Therefore, in the presence of a MV that is created by removing one sublattice atom $\left(A\right.$ or $B$ ), we have $N_{A} \neq N_{B}$ and thus the sublattice symmetry is broken. Since a DV can be created by removing two sublattice atoms ( $A, B$ or $A, A$ or $B, B)$, for the case of DV2 that is created by removing two $A$ (or two $B$ ) sublattice atoms, we have $N_{A} \neq N_{B}$, which breaks the sublattice symmetry of PNR; whereas for the case of DV1 that is created by removing both $A$ and $B$ sublattice atoms, we still have $N_{A}=N_{B}$, which preserves the sublattice symmetry of PNR.

In Fig. 3, we show the results for the cases of no-vacancy (NV), MV, DV1, and DV2 as indicated: Figs. 3(a) and 3(c) are for APNR and Figs. 3(b) and 3(d) are for ZPNR. Here, the ribbon length and width are set to $L=10 \mathrm{~nm}$ and $W=4$ $\mathrm{nm}$ for all the simulated PNRs. To see clearly the effect of a single vacancy on the DOS, the local DOS (LDOS) calculated at neighboring sites around the vacancy is superimposed on the DOS. It is clear that, for both APNR and ZPNR, introducing a single vacancy decreases the conductance value and smoothes the conductance step. As expected, we find that (i) the DV2 has a larger effect on the DOS and conductance than the MV, because a DV can be viewed as consisting of two MVs that are very close to each other and (ii) both the MV and DV2 have a larger impact on the DOS and conductance than the DV1, because the former two break the sublattice symmetry, while the latter one preserves such symmetry. These results are consistent with those previously found for graphene $[49,50]$. Note that all types of single vacancies (i.e., MV, DV1, and DV2) have little influence on the DOS of PNR outside the band gap (i.e., the bulk DOS of PNR), because the lattice region removed by a single vacancy is negligibly small as compared to the whole part of PNR. Nevertheless, they can affect the bulk and edge states of PNR via the vacancy scattering, and can thus affect the bulk and edge conductance of PNR.

For defective APNR, as shown in Figs. 3(a) and 3(c), both the MV and DV2 result in resonant peaks in the DOS within the band gap, which correspond to quasilocalized vacancy states. However, the DV1 does not give rise to such peaks because this two-atom vacancy preserves the sublattice symmetry of PNR (while such a symmetry is broken by both the MV and DV2). The results are very different for defective ZPNR, as shown in Figs. 3(b) and 3(d). We see that both the MV and DV2 induce resonant peaks in the edge DOS within the band gap, and simultaneously cause antiresonant dips in the edge conductance. The antiresonant dips in the edge conductance are mainly due to the vacancy scattering on the edge propagating modes. Intuitively, the vacancy located in the bulk center of ZPNR should not have a considerable influence on its edge conductance, because the vacancy position is far from the edges of ZPNR. To understand this counterintuitive result, we have to calculate the spatial LDOS of edge propagating modes of ZPNR and to examine how it is affected by a bulk central vacancy. The results are plotted in Figs. 3(e) and 3(f), corresponding to the cases of $\mathrm{NV}$ and $\mathrm{MV}$, respectively. From these two panels, we see that the quasilocalized vacancy state is considerably coupled to the edge state at the upper boundary of ZPNR, which may lead to a considerable edgevacancy scattering and thus an antiresonant dip in the edge 


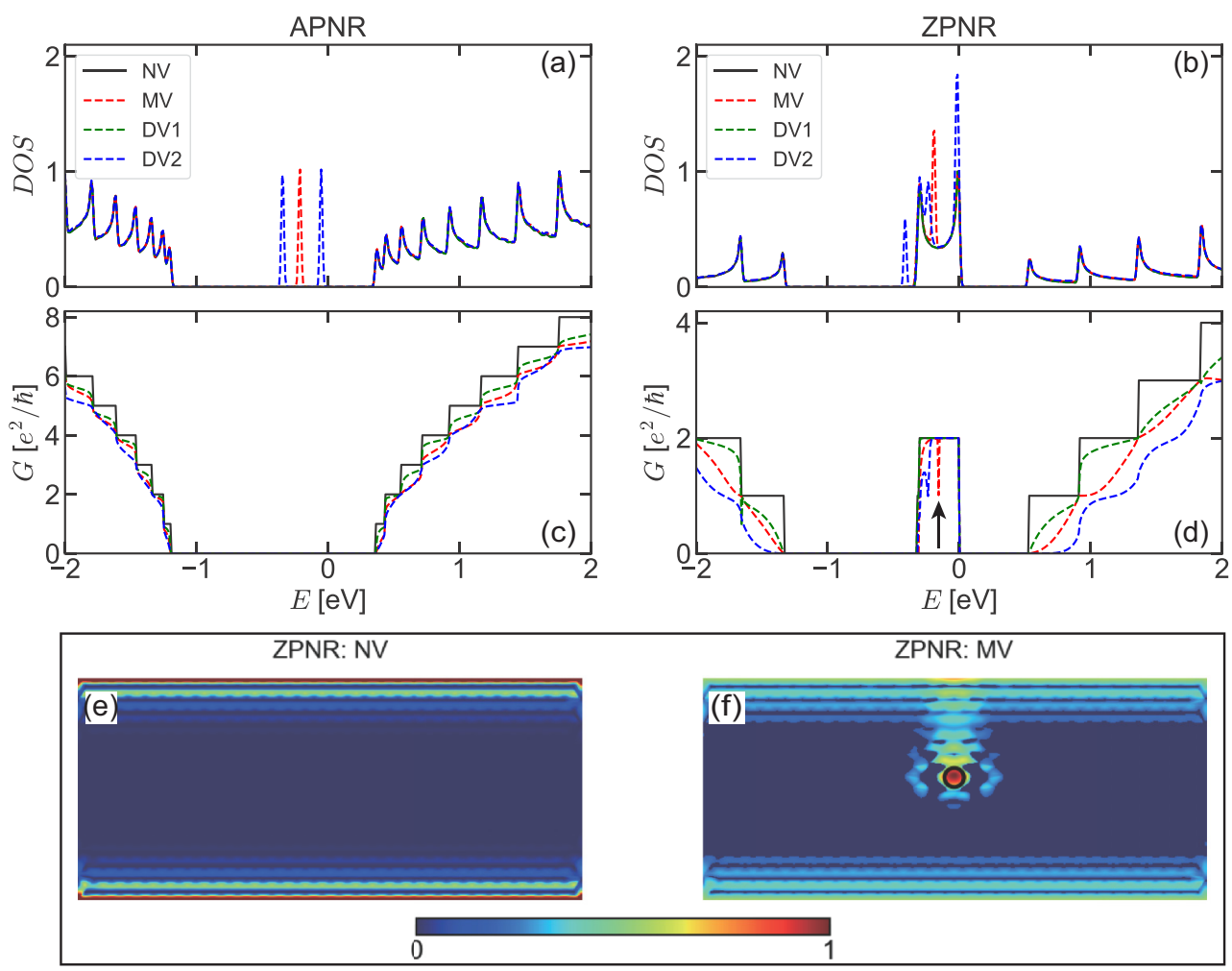

FIG. 3. (a)-(d) DOS and conductance of defective APNR and ZPNR for different types of a single vacancy placed in the center of the PNR, together with the results when no vacancy (NV) is present; (e) and (f) Spatial LDOS of edge propagating modes in the ZPNR without and with a single vacancy. Here, NV, MV, DV1, and DV2 denote the cases of no vacancy, monovacancy, divacancy of type I, and divacancy of type II, respectively. In panel $(\mathrm{d})$, the black arrow indicates the energy of those edge propagating modes at which their spatial LDOS is calculated. In panel (f), the black circle indicates the single vacancy and its position.

conductance. Note that there is an asymmetry in the coupling of the vacancy state with the edge state. This is because the quasilocalized vacancy state is spatially highly anisotropic, due to the inequivalent sublattices at which the vacancy is created. For instance, the spatial LDOS of the vacancy state shown in Fig. 3(f) is mostly distributed between the center and the upper edge of ZPNR. This anisotropic feature of the quasilocalized vacancy state was experimentally observed on the surface of BP [37]. We further find that with increasing ribbon width $W$, such a vacancy-edge coupling decreases (and even disappears) and consequently, the edge conductance becomes less affected (and even unaffected) by the bulk central vacancy.

Moreover, for defective ZPNR shown in Figs. 3(b) and 3(d), there is a one-to-one correspondence between the resonant peak(s) in the edge DOS and the antiresonant dip(s) in the edge conductance for the MV case, and those peak(s) and dip(s) have the same energy positions. But there is no such correspondence for the DV2 case, e.g., two resonant peaks in the edge DOS with only one of them giving rise to an antiresonant dip in the edge conductance. This is because the other peak is not located within the energy range of the edge DOS, although it is located within the band gap, and thus this peak has no influence on the edge conductance. Again, the DV1 does not give rise to such resonant peaks in the edge DOS, and thus the edge conductance is almost unaffected by this two-atom vacancy. Although MV and DV have distinctive influences on the edge conductance, they have almost the same effect on the bulk conductance, i.e., both of them cause a significant decrease of the conductance value.

Next we study the effect of the vacancy position on the DOS and conductance of defective APNR and ZPNR. Without loss of generality, we show the results for the MV case, because a DV can be viewed as two closely positioned MVs. In Fig. 4, we plot the DOS and conductance of defective APNR and ZPNR for different MV positions, together with the results of their perfect counterparts with no vacancy (NV). Here, the ribbon length and width are set to $L=10 \mathrm{~nm}$ and $W=4 \mathrm{~nm}$ for all simulated PNRs. The vacancy (MV) is assumed to move along the vertical central line of the scattering region (indicated by the red dashed line in Fig. 1) when changing its position, characterized by a vertical distance $d$ from the center of PNR. It can be clearly seen from this figure that the DOS and conductance of defective PNR depend sensitively on the MV position. For defective ZPNR, as shown in Figs. 4(b) and 4(d), by moving such vacancy from its center to its edge (i.e., changing $d$ from $0.2 \mathrm{~nm}$ to $1.6 \mathrm{~nm}$ ), the resonant peak(s) in the edge DOS as well as the antiresonant dip(s) in the edge conductance are both shifted in energy. This is not surprising for defective ZPNR because of the one-to-one correspondence between resonant peak(s) in the edge DOS and antiresonant $\operatorname{dip}(s)$ in the edge conductance. However, there is no such correspondence for defective APNR due to the absence of the in-gap (edge) conductance, as shown in Figs. 4(a) and 4(c), where the MV induces only a resonant peak in the DOS within 

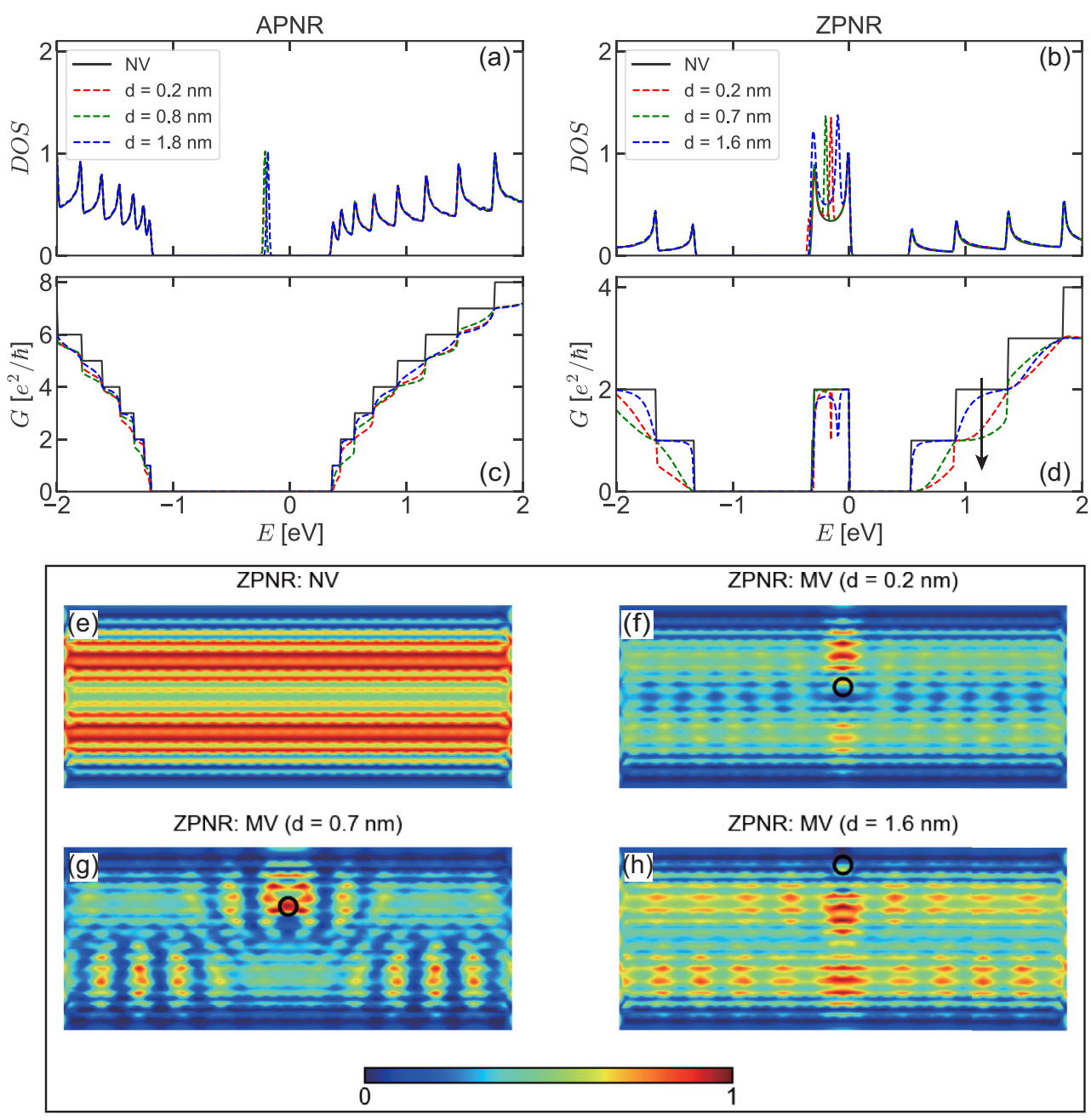

FIG. 4. (a)-(d) DOS and conductance of defective APNR and ZPNR for different positions $(d)$ of the monovacancy (MV) from the center of the PNR, together with the results of their perfect counterparts with no vacancy (NV). (e)-(h) Spatial LDOS of bulk propagating modes in the ZPNR for the cases of NV and MV. In panel (d), the black arrow indicates the energy of those bulk propagating modes at which their spatial LDOS is calculated. In panels (f)-(h), the black circles indicate the different vacancy positions.

the band gap, whose energy position is very slightly shifted by varying its position.

In addition to the inside-band-gap effects of the vacancy position (i.e., on the DOS and conductance of edge propagating modes), it is also interesting to look into its outside-band-gap effects (e.g., on the conductance of bulk propagating modes). As shown in Figs. 4(c) and 4(d), for the different conductance plateaus, changing the MV position gives rise to different consequences. For instance, the value of the first conductance plateau decreases continuously by moving the MV from the edge of ZPNR $(d=1.6 \mathrm{~nm})$ to the center of ZPNR $(d=$ $0.2 \mathrm{~nm}$ ). This result can be readily understood in an intuitive way. As the MV is moved closer to the center of ZPNR, it certainly affects the bulk conductance more significantly. However, for the second or higher conductance plateaus, the situation becomes somewhat complicated. The conductance value does not decrease continuously by moving the MV from the edge of ZPNR to the center of ZPNR, e.g., the central MV $(d=0.2 \mathrm{~nm})$ does not induce the largest decrease of the value of the second conductance plateau. Intuitively, this result is not easy to be understood. Again, one has to examine how the MV position affects the spatial LDOS of bulk propagating modes in the second conduction or valence subbands. The results are plotted in Figs. 4(e)-4(h), corresponding to the cases of NV, $\operatorname{MV}(d=0.2 \mathrm{~nm}), \operatorname{MV}(d=0.7 \mathrm{~nm})$, and $\operatorname{MV}(d=1.6 \mathrm{~nm})$, respectively. As can be seen from these four panels, the MV renders the bulk propagating modes to be less extended due to the vacancy scattering, which depends sensitively on the MV position. This may explain our counterintuitive result as to why the central MV ( $d=0.2 \mathrm{~nm})$ does not have the largest impact on the second conductance plateau. Similar results obtained for defective APNR can be understood in the same way.

\section{RANDOM DISTRIBUTION OF VACANCIES}

In this part, we turn to the effects of a random distribution of vacancies on the DOS and conductance of PNRs. Such vacancies can be used to simulate fabrication-induced and intrinsic (native) lattice defects in realistic PNR samples. Due to the randomness nature of such vacancies, we calculate the averaged properties by simulating many different defective PNRs for a given ribbon size and a given vacancy 

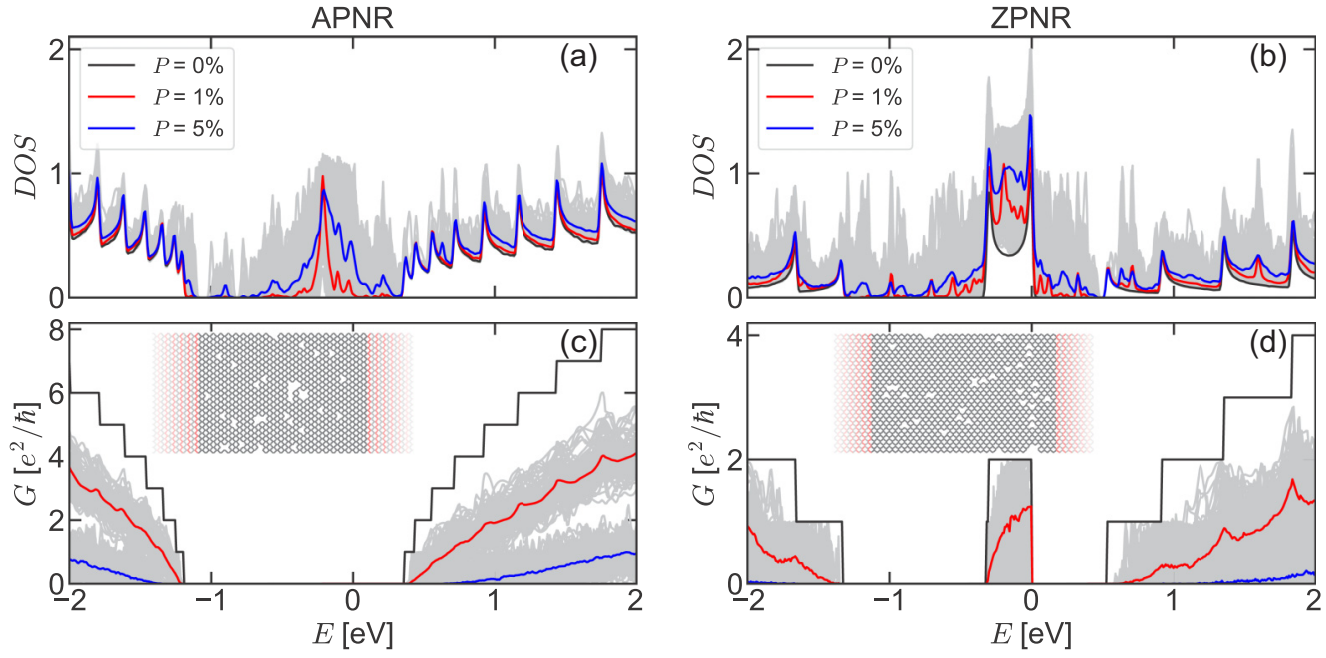

FIG. 5. DOS and conductance of defective PNRs with random vacancies for different vacancy concentrations $P$ as indicated, together with the results of their perfect counterparts with no vacancy $(P=0)$ : (a), (c) for the APNR case and (b), (d) for the ZPNR case. Here, each panel contains the individual results for all the simulated PNRs with vacancies (gray lines), the averaged results of them (color lines), and the result for perfect PNR (black line). The inset of panels (c) and (d) show schematically the APNR and ZPNR containing a random distribution of vacancies.

concentration. The number of simulated samples is chosen sufficiently large to decrease sample-to-sample fluctuations and to obtain converged results. It was shown previously [40] that converged results can be obtained for an ensemble of 50 to 200 random distributions, and, in the present paper, we took an ensemble of 100 random distributions.

Random vacancies in the simulated PNRs are implemented by randomly removing single atoms from both the edge and
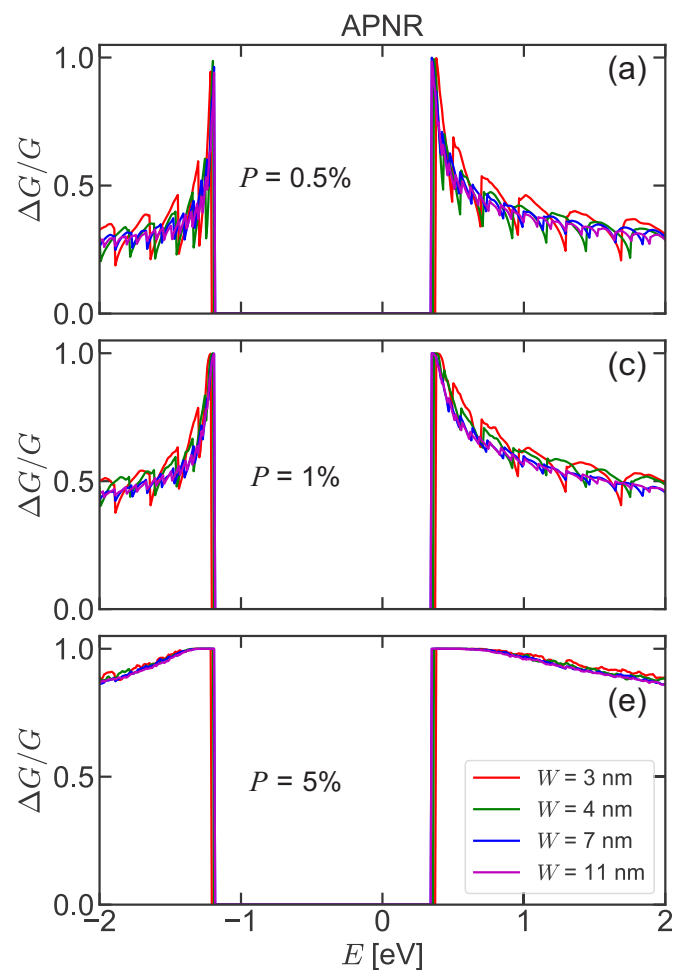

bulk regions of these PNRs. In Fig. 5, we show the effects of such vacancies on the DOS and conductance of APNR and ZPNR for different vacancy concentrations $P$ as indicated. Here, the ribbon length and width are set to be $L=10 \mathrm{~nm}$ and $W=4 \mathrm{~nm}$ for all the simulated samples, and the vacancy concentration $P$ is defined as the ratio of the number of removed atoms to the number of total atoms. As can be seen from this figure, with increasing vacancy concentration, the conductance

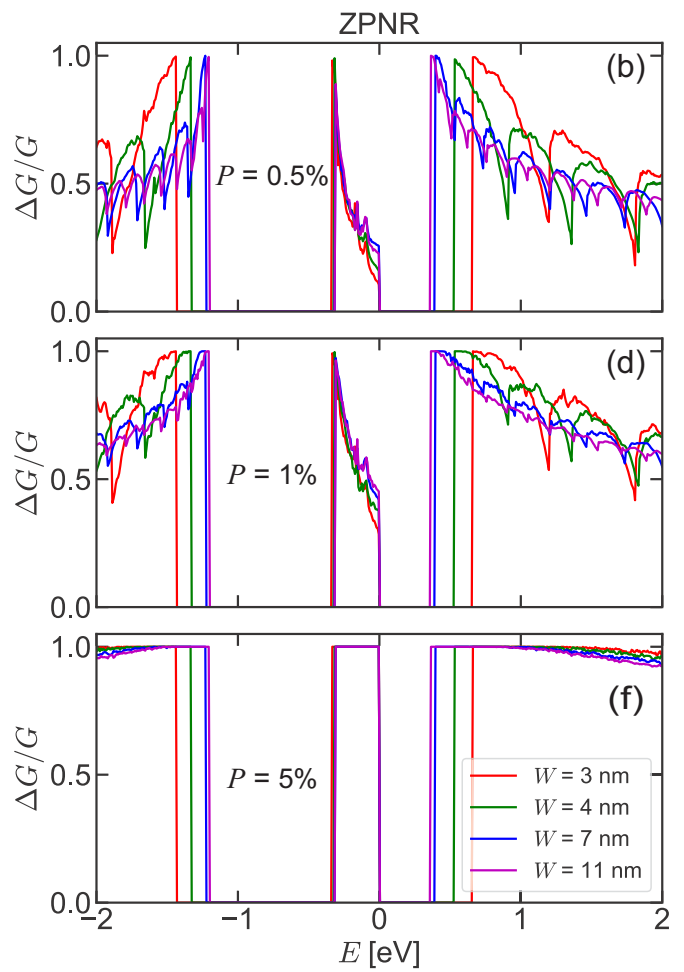

FIG. 6. Ratios of the change in the conductance of defective PNRs with random vacancies relative to that of their perfect counterparts, for different ribbon widths $W$ and vacancy concentrations $P$ as indicated: (a), (c), (e) for the APNR case and (b), (d), (f) for the ZPNR case. Here, the ribbon length is set to be $L=10 \mathrm{~nm}$ for all the simulated PNRs. 

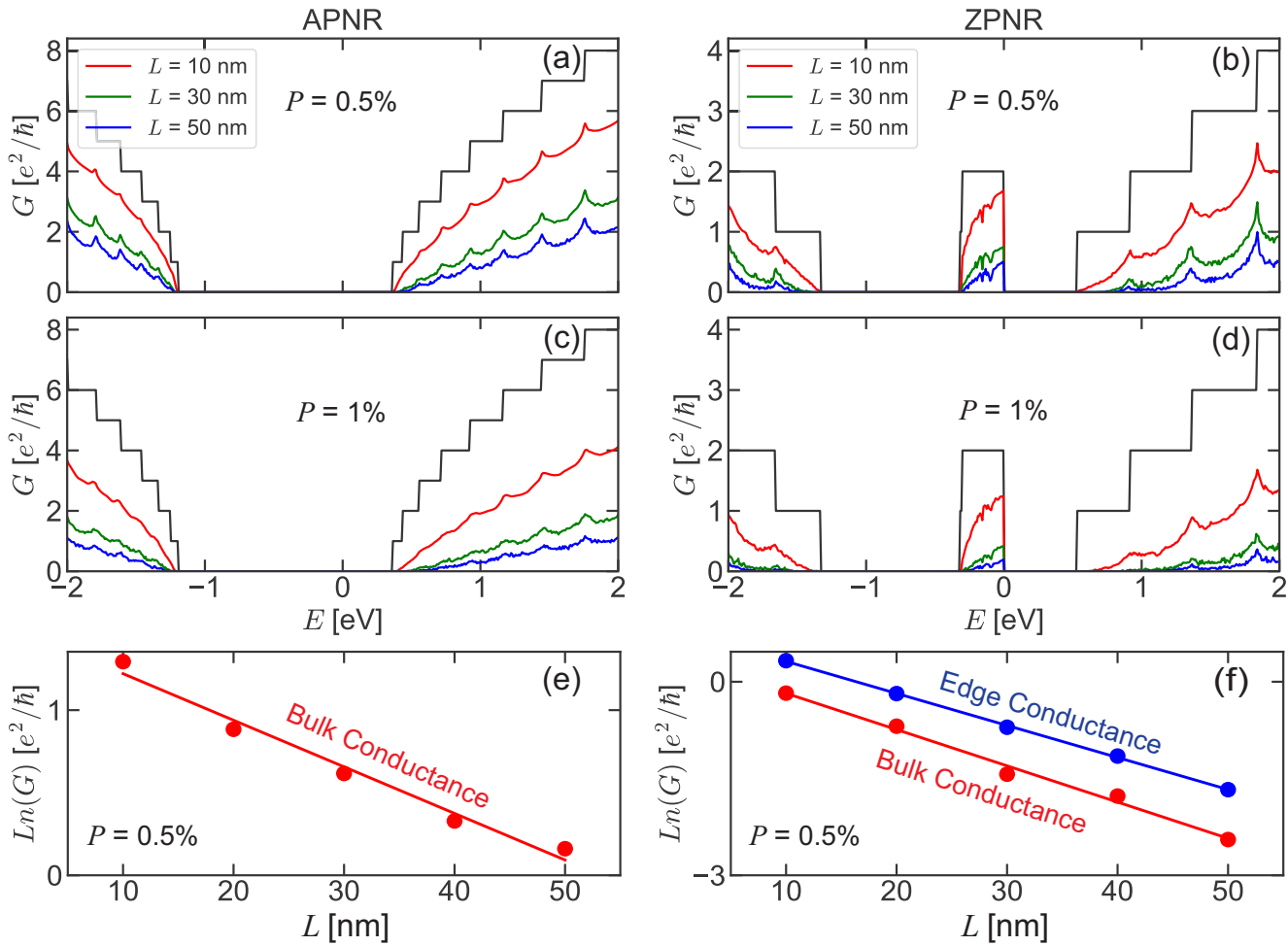

FIG. 7. Conductance of defective PNRs with random vacancies for different ribbon length $L$ and vacancy concentrations $P$ as indicated: (a), (c), (e) for the APNR case and (b), (d), (f) for the ZPNR case. Here, the ribbon width is set to $W=4$ nm for all the simulated PNRs. In panels (a)-(d), black lines are the results of perfect PNRs with no vacancy $(P=0)$, which are presented for reference purposes. In panels (e) and (f), red (blue) dots are the numerical results of bulk (edge) conductance, and corresponding lines are the fitted ones.

of both APNR and ZPNR is strongly suppressed, and no quantized plateaus are observed. For the APNR case, due to the strong decrease of the conductance, the transport gap increases from $1.52 \mathrm{eV}$ to $1.61 \mathrm{eV}$ and to $2.05 \mathrm{eV}$ with increasing $P$ from 0 to $1 \%$ and to $5 \%$. In particular, for the case of ZPNR, the edge conductance is fully suppressed as the vacancy concentration is increased to $P=5 \%$, leading to a global transport gap that is not present in perfect ZPNR. This indicates that with increasing vacancy concentration ZPNR will undergo a metalto-semiconductor transition. The physical reason behind this is that random vacancies act as randomly distributed short-range scatterers and induce very strong back scattering, leading to the so-called Anderson localization. Therefore, in the presence of random atomic vacancies and with increasing vacancy concentration, three different transport regimes, i.e., ballistic, diffusive, and Anderson localization, can clearly be observed in APNRs and ZPNRs, as described above.

We note that in the presence of a random distribution of vacancies, the DOS of both APNR and ZPNR is almost fully extended over the whole band gap, as shown in Figs. 5(a) and 5(b). This is due to the presence of many quasilocalized vacancy states that are overlapping among each other. For defective APNR, the presence of such vacancy states makes it difficult to extract the band gap from the DOS spectrum, as shown in Fig. 5(a). However, from the conductance spectrum it is possible to extract a distinct transport gap, as shown in Fig. 5(c).

As is known, ribbon length and width are important parameters in tuning the electronic, optical, and transport properties of PNRs. For instance, the fundamental band gap, the carrier effective mass, and the optical absorption coefficient of both APNR and ZPNR can be efficiently tuned by varying the ribbon width [23].

Here, to investigate the effects of ribbon length and width on the conductance of defective PNR with random vacancies, we introduce a physical quantity which characterizes the ratio of the change in the conductance of defective PNR relative to that of perfect one. Mathematically, this quantity is defined as $\Delta G / G=\left(G-G^{\prime}\right) / G$, with $G$ and $G^{\prime}$ being the conductance of perfect and defective PNRs, respectively. Thus, a larger value of $\Delta G / G$ means a higher sensitivity of PNR to vacancy disorder.

In Fig. 6, we show the relative change of the conductance of defective PNRs, $\Delta G / G$, for different ribbon widths $W$ and vacancy concentrations $P$ as indicated: Figs. 6(a), 6(c) and 6(e) for the APNR case and Figs. 6(b), 6(d) and 6(f) for the ZPNR case. Here, the ribbon length is fixed at $L=10 \mathrm{~nm}$ for all the simulated PNRs. From this figure, we find that (i) for fixed vacancy concentration, both narrower APNRs and ZPNRs are more sensitive to vacancy disorder than their wider counterparts, because vacancies are distributed more sparsely in the wider PNRs; (ii) for fixed ribbon width and vacancy concentration, ZPNRs are more sensitive to vacancy disorder than APNRs, because the carrier (electron or hole) effective mass of APNR is smaller than that of ZPNR, as can be verified from their band structures shown in Figs. 2(a) and 2(d), and therefore, the relaxation time (due to vacancy scattering) is longer for APNR than for ZPNR; and (iii) with increasing 
vacancy concentration, the difference between the sensitivities of APNR and ZPNR to vacancy disorder becomes smaller and smaller, and eventually disappears (i.e., $\Delta G / G=1$ ) for all the ribbon widths due to strong Anderson localization.

In Fig. 7, we show the conductance of defective PNRs with random vacancies for different ribbon lengths $L$ and vacancy concentrations $P$ as indicated: Figs. 7(a), 7(c) and 7(e) for the APNR case and Figs. 7(b), 7(d) and 7(f) for the ZPNR case. Here, the ribbon width is fixed at $W=4 \mathrm{~nm}$ for all the simulated PNRs. From this figure, we see that for fixed vacancy concentration, both longer APNRs and ZPNRs are more sensitive to vacancy disorder than their shorter counterparts. This result can be understood as follows: The conductance of defective PNR not only depends on the ribbon width $W$, which determines the number of available transport modes, but also depends on the ribbon length $L$, over which the vacancies are distributed. It is known that in a $1 \mathrm{D}$ system, the conductance decreases exponentially with the length of the system as $G=G_{0} \exp \left(-L / L_{0}\right)$, with $L_{0}$ being the localization length [51]. As shown in Figs. 7(e) and 7(f), our numerical simulations (indicated by color dots) agree with this prediction (indicated by color lines). However, the agreement is better for the edge conductance than the bulk conductance. This is because edge propagating modes are 1D transport channels as they are localized at the boundaries of PNR, while bulk propagating modes are mainly distributed in the bulk region of PNR and thus they are more 2D-like.

In addition, using the relation $G=G_{0} \exp \left(-L / L_{0}\right)$, we can fit out the localization length $L_{0}$ of defective PNR in the presence of vacancy disorder. For instance, from panels (e) and (f), the fitted localization lengths are $L_{0}=35.5 \mathrm{~nm}$ for the bulk conductance of APNR, $L_{0}=20.1 \mathrm{~nm}$ for the edge conductance of ZPNR, and $L_{0}=15.6 \mathrm{~nm}$ for the bulk conductance of ZPNR. Here, the values of $L_{0}$ are extracted at energy $E=1.2 \mathrm{eV}$ for the bulk conductance and at energy $E=-0.1 \mathrm{eV}$ for the edge conductance. Generally, this length is energy dependent and in transport experiments it can be measured around the Fermi energy. Moreover, our numerical calculations indicate that the localization length $L_{0}$ decreases for both the bulk and edge conductance with increasing vacancy concentration $P$, as expected.

\section{CONCLUDING REMARKS}

We have theoretically investigated the electronic and transport properties of defective PNRs containing atomic vacancies by means of the TB approach. Both cases of single and random vacancies were considered, and three types of single vacancies, i.e., monovacancy (MV), divacancy of type I (DV1), and divacancy of type II (DV2) were investigated. For comparative purposes, we also presented the results of perfect PNRs without vacancies.

We found that perfect APNRs and ZPNRs show very different electronic and transport properties. For instance, perfect ZPNRs exhibit a metallic behavior due to the presence of edge states within the band gap. These edge states are propagating along the zigzag boundaries, giving rise to nonzero conductance when the Fermi energy is located within the band gap. However, perfect APNRs have no such (edge) states and thus exhibit a semiconducting behavior.

For defective APNRs and ZPNRs, we found that single vacancies can create quasilocalized states and can thus affect their electronic and transport properties. Their DOS and conductance are found to depend sensitively on the vacancy type and on the vacancy position. For instance, MV and DV2 break the sublattice symmetry and have a larger impact on the DOS and conductance than DV1 that preserves such symmetry. Single vacancies like MV and DV2 cause resonant peaks in the DOS of both APNRs and ZPNRs, but simultaneously cause antiresonant dips in the edge conductance of ZPNRs. Those dips are induced by the scattering of edge modes by single vacancies because there is a considerable coupling between propagating edge states and quasilocalized vacancy states. By moving a single vacancy (e.g., MV) from the PNR center to the PNR edge, the conductance of bulk propagating modes are more strongly influences by an edge vacancy at higher energies. This is because those bulk modes have a considerable spatial LDOS close to the PNR edge and thus they can be more affected by the edge vacancy than by the central one.

In the presence of a random distribution of vacancies, the conductance of both APNRs and ZPNRs is strongly influenced. With increasing vacancy concentration, three different transport regimes, i.e., ballistic, diffusive, and Anderson localization, can clearly be distinguished. In particular, APNRs exhibit a lower conductance and a larger transport gap; while ZPNRs that are known to be metallic due to the presence of edge states become semiconducting: edge conductance is vanishing and transport gaps are induced due to Anderson localization. Moreover, we find that (i) for fixed vacancy concentration, both APNRs and ZPNRs of narrower width and/or longer length are more sensitive to vacancy disorder than their wider and/or shorter counterparts and (ii) for the same ribbon length and width, ZPNRs are more sensitive to vacancy disorder than APNRs. Our theoretical paper provides basic insights into potential applications of realistic PNRs in electronic transport devices.

\section{ACKNOWLEDGMENTS}

This work was financially supported by the Flemish Science Foundation (FWO-Vl), the FLAG-ERA TRANS 2D TMD, and by the Chinese Academy of Sciences (CAS).
[1] H. Liu, Y. Du, Y. Deng, and P. D. Ye, Chem. Soc. Rev. 44, 2732 (2015).

[2] L. Kou, C. Chen, and S. C. Smith, J. Phys. Chem. Lett. 6, 2794 (2015).
[3] A. H. Woomer, T. W. Farnsworth, J. Hu, R. A. Wells, C. L. Donley, and S. C. Warren, ACS Nano 9, 8869 (2015).

[4] A. Carvalho, M. Wang, X. Zhu, A. S. Rodin, H. Su, and A. H. C. Neto, Nat. Rev. Mater. 1, 16061 (2016). 
[5] L. Li, Y. Yu, G. J. Ye, Q. Ge, X. Ou, H. Wu, D. Feng, X. H. Chen, and Y. Zhang, Nat. Nanotechnol. 9, 372 (2014).

[6] H. Liu, A. T. Neal, Z. Zhu, Z. Luo, X. Xu, D. Tomanek, and P. D. Ye, ACS Nano 8, 4033 (2014).

[7] S. P. Koenig, R. A. Doganov, H. Schmidt, A. H. C. Neto, and B. Özyilmaz, Appl. Phys. Lett. 104, 103106 (2014).

[8] A. Castellanos-Gomez, L. Vicarelli, E. Prada, J. O. Island, K. L. Narasimha-Acharya, S. Blanter, D. J. Groenendijk, M. Buscema, G. A. Steele, J. V. Alvarez, H. W. Zandbergen, J. J. Palacios, and H. S. J. van der Zan, 2D Mater. 1, 025001 (2014).

[9] J. R. Brent, N. Savjani, E. A. Lewis, S. J. Haigh, D. J. Lewis, and P. O'Brien, Chem. Commun. 50, 13338 (2014).

[10] A. S. Rodin, A. Carvalho, and A. H. C. Neto, Phys. Rev. Lett. 112, 176801 (2014).

[11] J. Qiao, X. Kong, Z. X. Hu, F. Yang, and W. Ji, Nat. Commun. 5, 4475 (2014).

[12] F. Xia, H. Wang, and Y. Jia, Nat. Commun. 5, 4458 (2014).

[13] D. Çakır, H. Sahin, and F. M. Peeters, Phys. Rev. B 90, 205421 (2014).

[14] T. Low, R. Roldán, H. Wang, F. Xia, P. Avouris, L. M. Moreno, and F. Guinea, Phys. Rev. Lett. 113, 106802 (2014).

[15] S. Yuan, A. N. Rudenko, and M. I. Katsnelson, Phys. Rev. B 91, 115436 (2015).

[16] A. Jain and A. J. H. McGaughey, Sci. Rep. 5, 8501 (2015).

[17] Z. Luo, J. Maassen, Y. Deng, Y. Du, R. P. Garrelts, M. S. Lundstrom, P. D. Ye, and X. Xu, Nat. Commun. 6, 8572 (2015).

[18] A. Chaves, T. Low, P. Avouris, D. Çakır, and F. M. Peeters, Phys. Rev. B 91, 155311 (2015).

[19] R. Schuster, J. Trinckauf, C. Habenicht, M. Knupfer, and B. Büchner, Phys. Rev. Lett. 115, 026404 (2015).

[20] X. Wang, A. M. Jones, K. L. Seyler, V. Tran, Y. Jia, H. Zhao, H. Wang, L. Yang, X. Xu, and F. Xia, Nat. Nanotechnol. 10, 517 (2015).

[21] M. Y. Han, B. Özyilmaz, Y. Zhang, and P. Kim, Phys. Rev. Lett. 98, 206805 (2007).

[22] L. A. Ponomarenko, F. Schedin, M. I. Katsnelson, R. Yang, E. W. Hill, K. S. Novoselov, and A. K. Geim, Science 320, 356 (2008).

[23] V. Tran and L. Yang, Phys. Rev. B 89, 245407 (2014).

[24] A. Carvalho, A. S. Rodin, and A. H. Çastro Neto, Europhys. Lett. 108, 47005 (2014).

[25] M. Ezawa, New J. Phys. 16, 115004 (2014).

[26] Z. Zhu, C. Li, W. Yu, D. Chang, Q. Sun, and Y. Jia, Appl. Phys. Lett. 105, 113105 (2014).

[27] J. Zhang, H. J. Liu, L. Cheng, J. Wei, J. H. Liang, D. D. Fan, J. Shi, X. F. Tang, and Q. J. Zhang, Sci. Rep. 4, 6452 (2014).

[28] M. M. Grujić, M. Ezawa, M. Ž. Tadić, and F. M. Peeters, Phys. Rev. B 93, 245413 (2016).
[29] H. Guo, N. Lu, J. Dai, X. Wu, and X. C. Zeng, J. Phys. Chem. C 118, 14051 (2014).

[30] X. Y. Han, H. M. Stewart, S. A. Shevlin, C. R. A. Catlow, and Z. X. Guo, Nano Lett. 14, 4607 (2014).

[31] E. T. Sisakht, M. H. Zare, and F. Fazileh, Phys. Rev. B 91, 085409 (2015).

[32] R. Ma, H. Geng, W. Y. Deng, M. N. Chen, L. Sheng, and D. Y. Xing, Phys. Rev. B 94, 125410 (2016).

[33] W. Hu and J. Yang, J. Phys. Chem. C 119, 20474 (2015).

[34] V. Wang, Y. Kawazoe, and W. T. Geng, Phys. Rev. B 91, 045433 (2015).

[35] G. Wang, R. Pandey, and S. P. Karna, Appl. Phys. Lett. 106, 173104 (2015).

[36] Y. Cai, Q. Ke, G. Zhang, B. I. Yakobson, and Y. W. Zhang, J. Am. Chem. Soc. 138, 10199 (2017).

[37] B. Kiraly, N. Hauptmann, A. N. Rudenko, M. I. Katsnelson, and A. A. Khajetoorians, Nano Lett. 17, 3607 (2017).

[38] J. V. Riffle, C. Flynn, B. St. Laurent, C. A. Ayotte, C. A. Caputo, and S. M. Hollen, arXiv:1712.08491.

[39] Q. Wu, L. Shen, M. Yang, Y. Cai, Z. Huang, and Y. P. Feng, Phys. Rev. B 92, 035436 (2015).

[40] M. Poojak and T. Suligoj, Nano Res. 9, 1723 (2016).

[41] C. Freysoldt, B. Grabowski, T. Hickel, J. Neugebauer, G. Kresse, A. Janotti, and C. G. Van de Walle, Rev. Mod. Phys. 86, 253 (2014).

[42] J. Hong, Z. Hu, M. Probert, K. Li, D. Lv, X. Yang, L. Gu, N. Mao, Q. Feng, L. Xie, J. Zhang, D. Wu, Z. Zhang, C. Jin, W. Ji, X. Zhang, J. Yuan, and Z. Zhang, Nat. Commun. 6, 6293 (2015).

[43] P. Vancsó, G. Z. Magda, J. Petô, J.-Y. Noh, Y.-S. Kim, C. Hwang, L. P. Biró, and L. Tapasztó, Sci. Rep. 6, 29726 (2016).

[44] A. N. Rudenko and M. I. Katsnelson, Phys. Rev. B 89, 201408(R) (2014).

[45] S. Datta, Electronic Transport in Mesoscopic Systems (Cambridge University Press, Cambridge, 1995).

[46] M. Wimmer, Quantum Transport in Nanostructures: From Computational Concepts to Spintronics in Graphene and Magnetic Tunnel Junctions, Ph.D. thesis, Universität Regensburg, 2009.

[47] C. W. Groth, M. Wimmer, A. R. Akhmerov, and X. Waintal, New J. Phys. 16, 063065 (2014).

[48] D. Moldovan and F. M. Peeters, Pybinding: A Python Package for Tight-Binding Calculations, doi:10.5281/zenodo.56818.

[49] T. C. Li and S.-P. Lu, Phys. Rev. B 77, 085408 (2008).

[50] M. D. Petrović and F. M. Peeters, Phys. Rev. B 94, 235413 (2016).

[51] P. A. Lee and T. V. Ramakrishnan, Rev. Mod. Phys. 57, 287 (1985). 